\title{
Improving a Self-curing Dental Resin by Eliminating Oxygen, Hydroquinone and Water from its Curing Process
}

\author{
En-Sheng KEH, Iwao HAYAKAWA, Hidekazu TAKAHASHI ${ }^{1}$, \\ Akihiko WATANABE ${ }^{2}$, Yasuhiko IWASAKI ${ }^{2}$, Kazunari AKIYOSHI ${ }^{2}$ \\ and Nobuo NAKABAYASHI ${ }^{2}$ \\ Complete Denture Prosthodontics, Department of \\ Masticatory Function Rehabilitation, \\ Division of Oral Health Science, Graduate School \\ ${ }^{1}$ Advanced Biomaterials, Department of Restorative Science, \\ Division of Oral Health Science, Graduate School \\ ${ }^{2}$ Department of Organic Material, Institute of \\ Biomaterials and Bioengineering \\ Tokyo Medical and Dental University \\ 1-5-4 Yushima, Bunkyo-ku, Tokyo 113-8549, Japan
}

Received July 15, 2002/Accepted September 30, 2002

\begin{abstract}
Self-curing dental resins are always manipulated in the presence of curing inhibitory factors such as oxygen, hydroquinone, water and another contaminants such as saliva and blood. The purpose of this study was to elucidate the effects of eliminating these curing inhibitory factors on resin properties. Several clinically relevant characteristics of an experimental resin cured in the absence of inhibitory factors (purified group) were determined and compared with the resin cured conventionally (control group). The purified group showed a significantly shorter induction period and higher reaction exotherm. It also showed significantly higher tensile strength and lower strain to failure. With regard to the amount of residual monomer, fracture toughness and fatigue strength the improvements were not significant. These findings suggest that the polymerization outcomes and mechanical properties of the purified group resin can be improved in general. Dental clinicians should pay attention to the storage and curing environments while manipulating the self-curing resin.
\end{abstract}

Key words: Self-curing resin, Polymerization, Mechanical property

\section{INTRODUCTION}

Self-curing dental resins have been widely used as materials for pouring-type dentures, denture lining/repairing and temporary restorations. They are always supplied as powder and liquid components. In the majority of commercial products, the powder component includes pre-polymerized polymer beads and an initiator (such as benzoyl peroxide; BPO); the liquid component contains the monomer and a chemical activator (such as $\mathrm{N}, \mathrm{N}$ - dimethyl-p-toluidine; DMPT). When the two components are mixed, DMPT provokes BPO to decompose into free radicals. The polymerization reaction of the monomer in liquid will be initiated and propagated at room temperature. For yielding optimal properties of self-curing resin, the monomer should come into intimate contact with beads, deeply diffuse into them and then be 
polymerized thoroughly in situ.

Since all the mixing and curing procedures are always performed in an ambient atmosphere and the patient's oral cavity, many curing inhibitory factors are expected to be encountered in daily dental practice. These factors include: (1) oxygen in ambient atmosphere and dissolved in the monomer ${ }^{1)}$, (2) water adsorbed on the bead's surface, (3) hydroquinone, which is usually used as a stabilizer in commercial products to prevent the unwanted polymerization during shipment and storage.

While manipulating the self-curing resin, free radicals easily react with oxygen in air. The resultant products, namely peroxide radicals, have very low reactivity to another monomer molecule that accounts for further polymerization reactions. This results in a slowdown of the overall reaction rate and incomplete polymerization. Peroxide radicals also introduce oxygen molecules into the polymer bulk. In this case, the peroxide-methacrylate co-oligomer will be formed. This is an unstable product and easily decomposed to formaldehyde and methyl pyruvate ${ }^{2)}$. Although water molecules do not interfere with the polymerization reaction directly, they might be adsorbed onto the bead's surface. The bead's wettability to the monomer will be compromised. Some bead-matrix interfacial defects might be left after being polymerized. While being subjected to mastication forces, these microscopic defects act as stress concentrators and eventually reduce the mechanical properties of the prosthesis. Owing to incompletely polymerization and the existence of defects, the mechanical properties would be markedly reduced. The curing process performed in dental clinics is more or less hindered by oxygen and water. It is suggested that the properties of dental resin will be improved by eliminating curing inhibitory factors. However, only a few studies have been reported ${ }^{3)}$.

The heat-releasing behavior provides essential information to clinicians for manipulating the self-curing resin properly. Differential scanning calorimetry (DSC) is a sensitive tool to monitor the heat released from the polymerization reaction ${ }^{4)}$. Since reaction heat directly corresponds to the monomer conversion, it has been used to estimate the extent of polymerization of dental resins ${ }^{5)}$. The residual monomer will cause mucosal irritation and deteriorate the mechanical properties of the polymer by acting as a plasticizer. Mechanical properties such as the tensile and fatigue strengths, the elastic modulus and the fracture toughness also provide clinically relevant information.

The purpose of this study was to elucidate the effects of eliminating these curing inhibitory factors on the property of an experimental self-curing resin. The heatreleasing behavior, quantity of residual monomer, tensile strength, elastic modulus, fracture toughness and fatigue strength of the resin cured in the absence of oxygen, water and hydroquinone (purified group) were determined and compared with those of resin cured by the method practiced daily (control group). 


\section{MATERIALS AND METHODS}

\section{Materials}

The compositions of a self-curing dental resin used in this study are listed in Table 1. The control and purified groups consisted of the same compositions, except the powder component of the latter was vacuum dried in a desiccator for 7 days to remove the adsorbed oxygen and water on the beads surface. The water content of powder can be reduced to one-ninth by this method $^{3)}$. In addition, the liquid was purified by adding $\mathrm{CaH}_{2}$ (Lot M0M9891, Nacalai Tesque, Kyoto, Japan) as a dehydrating agent, then distilled at $101^{\circ} \mathrm{C}$ at a pressure of $760 \mathrm{mmHg}$ to remove hydroquinone and water. The middle fraction of distilled MMA was collected and added DMPT thereafter. The powder and liquid were mixed in a weight ratio of $2: 1$ throughout this study. All the mixing, packing and curing procedures of the purified group were performed in an argon-filled chamber. Before being subjected to mechanical testing, all specimens were stored in a dark laboratory environment at $25^{\circ} \mathrm{C}$ for 48 hours.

\section{Heat-releasing behavior monitored by DSC}

After mixing powder and liquid for 30 seconds, $70 \mathrm{mg}$ of this uniform mixture was transferred to an aluminum sample container and tightly sealed immediately. The container was placed in a differential scanning calorimeter (DSC 100, Seiko, Tokyo, Japan). It was scanned isothermally at $25^{\circ} \mathrm{C}$ for 40 minutes. Five mixtures of each group were prepared and measured. Several curing process parameters described in this study were derived from DSC thermograms; curing initiating time $\left(\mathrm{T}_{\mathrm{i}}\right)$ : the exothermic peak starting to rise from the baseline; peak time $\left(\mathrm{T}_{\mathrm{p}}\right)$ : climax of the peak; curing termination time $\left(\mathrm{T}_{\mathrm{f}}\right)$ : while peak declining to the same level as the baseline; evolved heat: $(\Delta \mathrm{H} ; \mathrm{J} / \mathrm{g})$ which was calculated from the area under the exothermic peak (between $\mathrm{T}_{\mathrm{i}}$ and $\mathrm{T}_{\mathrm{p}}$ ) and the weight of each mixture.

\section{Tensile test}

A dumbbell specimen with a parallel portion $(2 \mathrm{~mm} \times 2 \mathrm{~mm} \times 15 \mathrm{~mm})$ was fabricated using a stainless steel mold. Resin dough ( 6 minutes after start of mixing at $25^{\circ} \mathrm{C}$ ) was packed into the mold then compressed with two glass-plates. After the dough was cured (60 minutes after packing), resin fins existing around the specimen border were carved using a sharp knife without further polishing. Six specimens were exam-

Table 1 Compositions of the self-curing dental resins used in this study

\begin{tabular}{lllll}
\hline Component & \multicolumn{1}{c}{ Composition } & Abbreviation & \multicolumn{1}{c}{ Manufacturer } & Lot No. \\
\hline Powder & Poly (MMA-co-EMA) beads & Poly (MMA-co- & Fujikura Kasei, Tokyo, Japan & PM-12 \\
& $(\mathrm{w} / \mathrm{w}=80: 20$; average diameter: $52.1 \mu \mathrm{m})$ & EMA $)$ & & W0K20B1 \\
benzoyl peroxide, 0.5wt $\%$ & BPO & Nacalai Tesque, Kyoto, Japan & M6P9258 \\
Liquid & methyl methacrylate & MMA & Kanto Chemical, Tokyo, Japan & $25289-01$ \\
& $\mathrm{~N}, \mathrm{~N}$ - dimethyl-p-toluidine, 0.5wt $\%$ & DMPT & Tokyo Kasei, Tokyo, Japan & FGH01 \\
\hline
\end{tabular}

Powder and liquid are mixed at a weight ratio of $2: 1$. 
ined using a testing machine (Model 1123, Instron, Canton, MA, USA) at a crosshead speed of $1 \mathrm{~mm} / \mathrm{min}$. The fracture surfaces of test specimens were observed with a scanning electronic microscope (JSM-5400, JEOL, Tokyo, Japan). Another five specimens were used to determine the strain to fracture and elastic modulus using a strain gauge (extensometer, No.2630-028) at the same tensile test parameters.

\section{Residual monomer measurement}

Four dumbbell specimens were prepared according to the above-mentioned manner. The amount of unreacted MMA in each specimen was determined with the high performance liquid chromatography (HPLC) method described in ISO Standard 1567: 1997. Each specimen (about $200 \mathrm{mg} ; 48$ hours after curing) was dissolved in $10 \mathrm{~mL}$ of tetrahydrofurane (THF) and stirred for 24 hours at room temperature. Then 8 $\mathrm{mL}$ of methanol was added to a $2 \mathrm{~mL}$ aliquot of the sample solution to precipitate the dissolved polymer. Five milliliters of the polymer and monomer containing slurry was centrifuged at $3,000 \mathrm{rpm}$ for 15 minutes. Fifty microliters of the supernatant solution was injected into an HPLC system (CCPE, Tosoh, Tokyo, Japan) equipped with a reversed-phase column (TSK-GEL CN-80-Ts, Tosoh, Tokyo, Japan; $4.6 \mu \mathrm{m} \times 250 \mathrm{~mm}$ ) at a flow rate as $0.5 \mathrm{~mL} / \mathrm{min}$ with the mobile phase of methanol/ water $(6 / 4 ; \mathrm{v} / \mathrm{v})$. The detector (UV-8010, Tosoh, Tokyo, Japan) was set for ultraviolet absorption at $229 \mathrm{~nm}$. The quantity of MMA in the sample solution was determined using a linear regression equation obtained from a calibration graph. Finally, the residual monomer was expressed as the weight percentage to each test specimen.

Fracture toughness $\left(K_{I C}\right)$ test

Fracture toughness was determined according to the ASTM Standard E- $399^{6)}$. Six bar-shaped specimens $(2 \mathrm{~mm} \times 5 \mathrm{~mm} \times 25 \mathrm{~mm}$ with a $2.5 \mathrm{~mm}$ deep notch $)$ for each group were prepared using a stainless steel mold inserted with a razor blade. The single-notch specimen was loaded on a 20 -mm wide span, and subjected to a threepoint bending test at a crosshead speed of $0.13 \mathrm{~mm} / \mathrm{min}$ using the testing machine mentioned previously. Fracture toughness $\left(K_{\mathrm{IC}}\right)$ was calculated using the following equation:

$$
K_{I C}=\frac{P \cdot S}{B \cdot W^{3 / 2}} \cdot F(\alpha) ; F(\alpha)=\frac{3 \alpha^{1 / 2}\left[1.99-\alpha(1-\alpha)\left(2.15-3.93 \alpha+2.7 \alpha^{2}\right)\right]}{2(1+2 \alpha)(1-\alpha)^{3 / 2}}
$$

where, $\mathrm{P}$ is the fracture load, $\mathrm{S}$ the span length, B the thickness, W the width, and $\alpha$ the ratio of the notch length to $\mathrm{W}$. The fracture surfaces were also observed by SEM.

\section{Fatigue test}

The fatigue strength at $10^{5}$ cycles was determined by the staircase method. Its detailed procedure was described elsewhere ${ }^{7)}$. The fatigue strength indicates that under such stress level the probability of specimen fracture before $10^{5}$ cycles is $50 \%$. Twenty dumbbell specimens for each group were subjected to a fatigue testing system 
(Model 8871, Instron, Canton, MA, USA) in sequence. Cyclic tensile stress was applied in sinusoidal shaped waves at $5 \mathrm{~Hz}$. The stress ratio was 0.1 . In this study, the initial stress level was $34 \mathrm{MPa}$ and stress increment was $2 \mathrm{MPa}$.

\section{Statistics analysis}

The results of the control and purified groups for each test were compared with Student's $t$ test. The statistical significance was set at the 0.05 probability level.

\section{RESULTS}

A representative DSC thermogram is shown in Fig. 1. The values of $T_{i}, T_{p}$ and $T_{f}$ obtained from each thermogram should be added with the time consumed for mixing, weighing and transferring the mixture sample to the DSC instrument. Each sample required about $2 \sim 3$ minutes before starting the DSC measurements. The derived curing process parameters are listed in Table 2. It was found that the purified group started to release reaction heat about 4 minutes faster than the control group. It also required 2 minutes shorter to reach the $\mathrm{T}_{\mathrm{p}}$. The purified group also showed

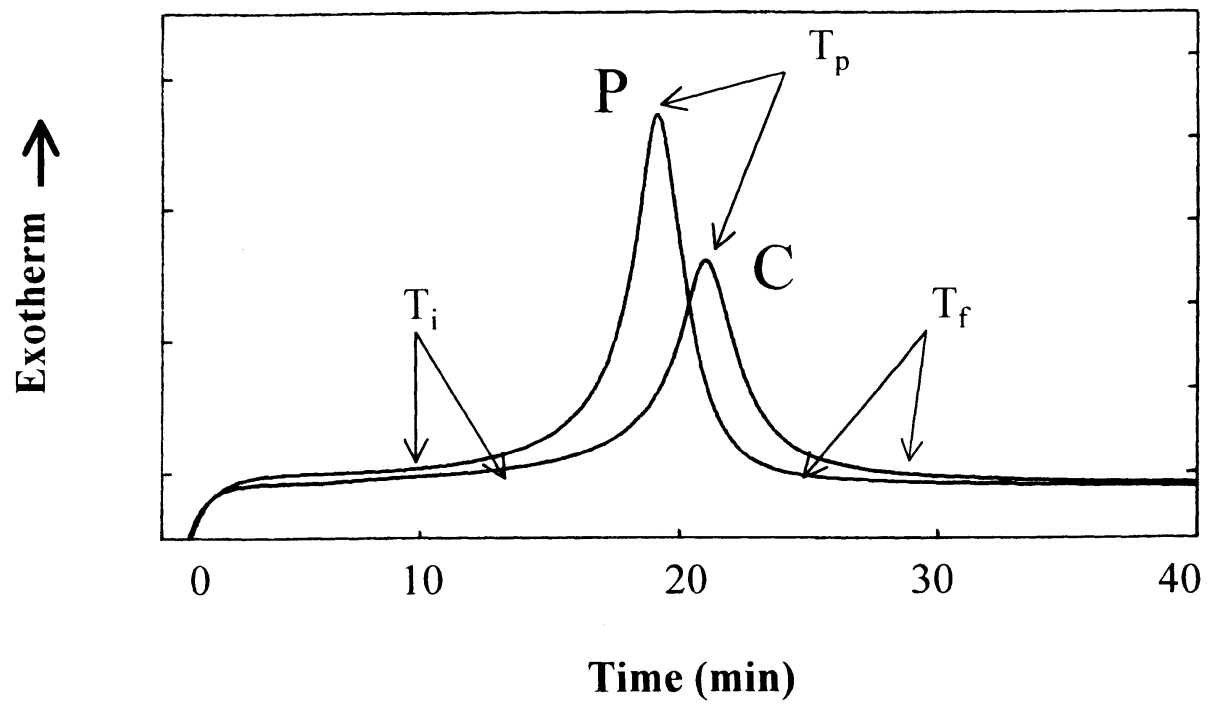

Fig. 1 The representative DSC thermograms.

Table 2 Curing process parameters of the control and purified groups. These values were counted from the start of mixing the powder and liquid

\begin{tabular}{ccccc}
\hline Group & $\mathrm{T}_{\mathrm{i}}(\min )$ & $\mathrm{T}_{\mathrm{p}}(\min )$ & $\mathrm{T}_{\mathrm{f}}(\min )$ & $\Delta \mathrm{H}(\mathrm{J} / \mathrm{g})$ \\
\hline Control & $15.6 \pm 1.5^{*}$ & $24.4 \pm 1.3^{*}$ & $33.8 \pm 1.4^{*}$ & $60.3 \pm 4.0^{*}$ \\
Purified & $11.4 \pm 0.9$ & $22.0 \pm 1.0$ & $28.6 \pm 1.6$ & $68.6 \pm 0.4$ \\
\hline
\end{tabular}

$\mathrm{n}=5$, Values are means $\pm \mathrm{SD} ;{ }^{*}$ indicates a significant difference between two groups $(\mathrm{p}<0.05)$ 
more reaction exotherm $(68.6$ vs $60.3 \mathrm{~J} / \mathrm{g})$. This suggests that the curing process of purified group was more easily initiated and effectively performed.

The amount of residual monomer and the mechanical properties are summarized in Table 3. The amount of residual monomer for the purified group was reduced (from 6.53 to $4.46 \%$ ), but the difference was not significant. In the results of the tensile test, the purified group showed significantly greater tensile strength $(40.2 \pm$ 0.4 us $36.7 \pm 1.2 \mathrm{MPa}$, increased by $10 \%$ ). The strain to fracture was significantly decreased from 26.4 to $18.1 \%$. The elastic modulus was also slightly increased but not significantly. The fracture surface of the purified group was predominant in smooth surfaces (Fig.2). On the other hand, the control group showed irregular surfaces with naked beads and craters after the beads detached, which represented the poor bead-matrix interfacial bonding. The $\mathrm{K}_{\mathrm{IC}}$ value of the purified group was slightly improved (from 1.50 to $1.65 \mathrm{MPa} \cdot \mathrm{m}^{1 / 2}$ ) but without significance. Their fracture surfaces did not show a marked difference (Fig. 3).

The staircase diagrams of the fatigue test are shown in Fig. 4. All test specimens showed plastic elongation after testing, especially the specimens subjected to the low stress amplitude and large cycles number. The fatigue strength of the purified group $(31.4 \pm 1.3 \mathrm{MPa})$ was slightly greater than that of the control group (30.4 $\pm 2.1 \mathrm{MPa})$. In contrast to the static tensile strength, the tensile fatigue strengths

Table 3 Results of residual monomor mcasurements, the tensile test, fracture toughness test and fatigue test for the control and purified groups

\begin{tabular}{lcccccc}
\hline Group & $\begin{array}{c}\text { Residual monomer } \\
(\mathrm{wt} \%), \mathrm{n}=4\end{array}$ & $\begin{array}{c}\text { Tensile strength } \\
(\mathrm{MPa}), \mathrm{n}=6\end{array}$ & $\begin{array}{c}\text { Strain at fracture } \\
(\%), \mathrm{n}=5\end{array}$ & $\begin{array}{c}\text { Elastic modulus } \\
(\mathrm{GPa}), \mathrm{n}=5\end{array}$ & $\begin{array}{c}\text { Fracture toughness } \\
\left(\mathrm{MPa} \cdot \mathrm{m}^{1 / 2}\right), \mathrm{n}=6\end{array}$ & $\begin{array}{c}\text { Fatigue strength } \\
(\mathrm{MPa}), \mathrm{n}=20\end{array}$ \\
\hline Control & $6.53 \pm 1.39$ & $36.7 \pm 1.6^{*}$ & $26.4 \pm 5.7^{*}$ & $2.59 \pm 0.65$ & $1.50 \pm 0.09$ & $30.4 \pm 2.1$ \\
Purified & $4.46 \pm 1.63$ & $40.2 \pm 0.4$ & $18.1 \pm 6.2$ & $3.43 \pm 0.72$ & $1.65 \pm 0.20$ & $31.4 \pm 1.3$ \\
\hline
\end{tabular}

Values are means $\pm S D$; ${ }^{*}$ indicates a significant difference between two groups $(p<0.05)$
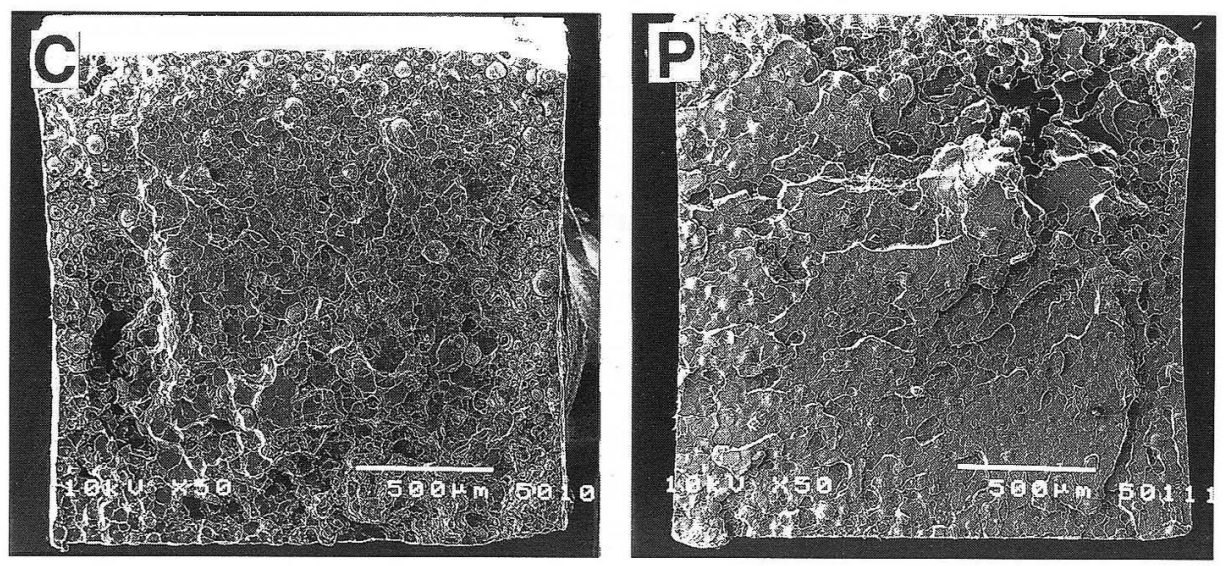

Fig. 2 Representative SEM $(\times 50)$ pictures of the fracture surface after tensile testing. 

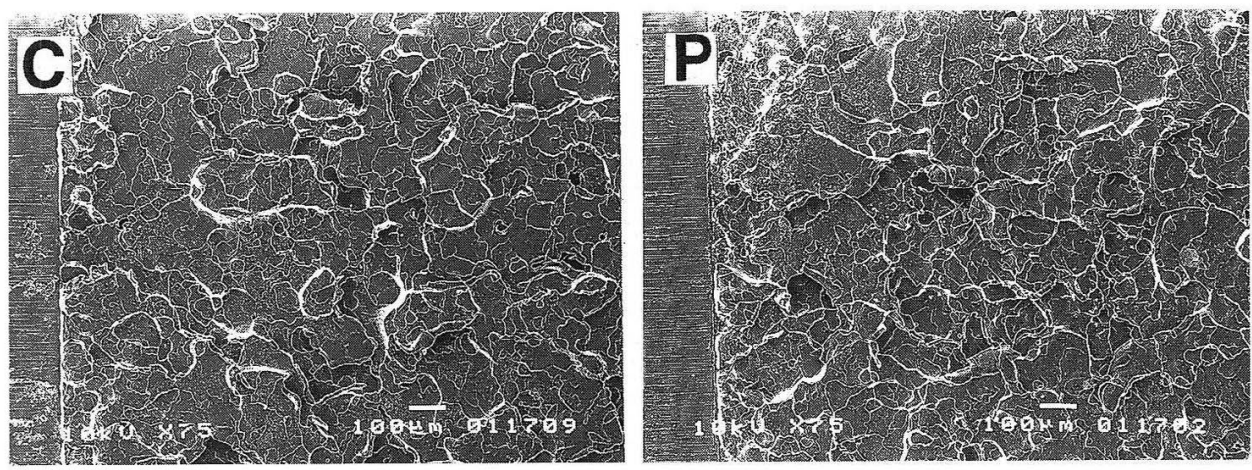

Fig. 3 Representative SEM $(\times 75)$ pictures of the fracture surface after fracture toughness testing.

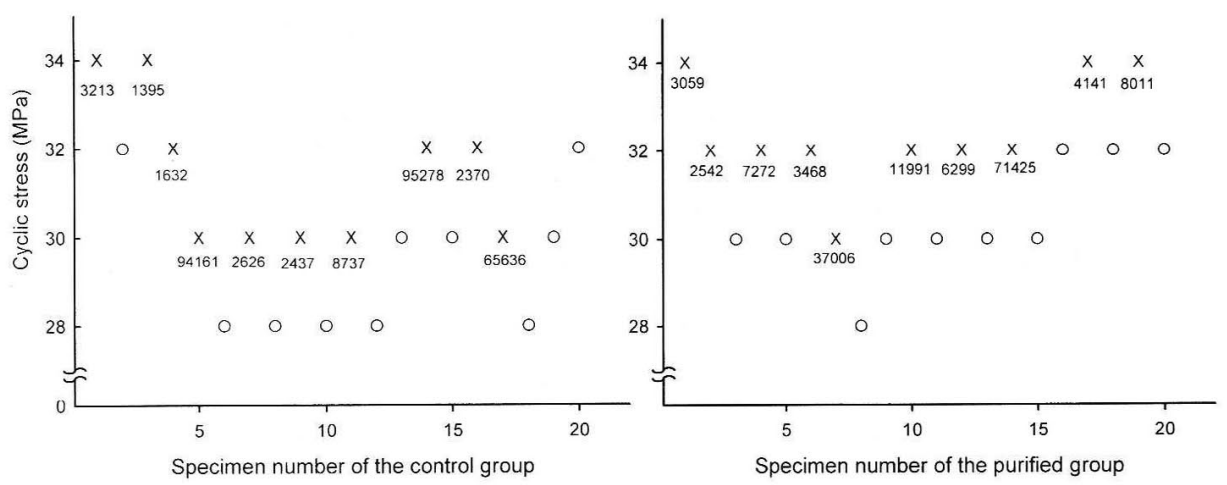

Fig. 4 Staircase diagrams show the results of the fatigue test at $10^{5}$ cycles.

of two groups were not significantly different.

\section{DISCUSSION}

The curing process of a self-curing resin is the combination of the free radical polymerization reaction and the physical change of the powder-liquid mixture. For eliminating oxygen, hydroquinone was expected to be advantageous to the polymerization reaction of the monomer. Although free radicals could be generated immediately after mixing, the so called "induction period" was observed in the DSC thermogram. Its duration is denoted by the time interval from the start of mixing until the $\mathrm{T}_{\mathrm{i}}$. Even if to a small extent of the polymerization reaction might be initiated and propagated, the released heat was not detected by DSC during this period. The longer induction period of the control group suggests that oxygen and hydroquinone consumed a significant amount of free radicals. Insufficient free radicals may also cause a lower conversion rate. This can explain why the control group 
showed $90 \%$ of the reaction heat of the purified group.

The mechanical properties of the resin bulk depend on the intrinsic properties of pre-polymerized beads, newly polymerized polymer (matrix) and bead-matrix interfacial characteristics. Although the larger amounts of free radicals can improve the outcome of the polymerization reaction in the purified group, it also unfortunately reduced the monomer diffusion time. The curing process of the purified group terminated 5 minutes earlier than the control group $(28.6$ us $33.8 \mathrm{~min}$ indicated as $\mathrm{T}_{\mathrm{f}}$ ). The penetrating depth of monomer molecules into the pre-polymerized beads might be limited. At the same time, less beads could be dissolved by monomer. Therefore, it was doubted whether the decreasing diffusion time should impair interfacial characteristics because bead-matrix interfacial failure might occur in such an area, where the polymer chains of matrix and beads are not deeply and tightly entangled. However, the fracture surface of the purified group after the present tensile test did not show interfacial debonding (Fig. 2). In contrast, the fracture surface of the control group showed weak interfacial bonding. These findings suggest that good wetting and more effective matrix polymerization might compensate for the inadequate monomer diffusion of the purified group. Good wetting and effective polymerization also caused the greater strength, greater modulus and lower strain; i.e., the higher deformation-resistance to tensile stress.

For gaining insight into the interfacial debonding under tensile stress, the fracture toughness test was conducted with the single-edge notched specimen. The $\mathrm{K}_{\mathrm{IC}}$ value indicates the ability of materials to resist crack propagation in a tensile opening mode. Although the ASTM Standard E-399 was originally applied to metallic materials, it has been widely used to determine the fracture toughness of composite $\operatorname{resin}^{8)}$ and bone cement ${ }^{9)}$. Due to the ductility of PMMA, especially in the presence of residual monomer and under a low crosshead speed $(0.13 \mathrm{~mm} / \mathrm{min})$, plastic deformation adjacent to the crack tip might be apparent. Therefore, the $\mathrm{K}_{\mathrm{IC}}$ data in this study have to be considered as the relative values only for comparative purposes. The crack propagation in front of the single-edged notch of the purified group was supposedly restricted due to the decrease in interfacial defects. However, $K_{\text {IC }}$ in this study was not significantly improved. The SEM pictures also did not show marked differences. Factors other than interfacial defects might govern the crack propagation phenomenon under the present $\mathrm{K}_{\mathrm{IC}}$ testing conditions.

Realizing the fatigue behavior of prosthetic structures that are subjected to continuous cyclic stress is essential for dental clinicians. Since there was no prior information on the fatigue behavior of this resin, a timesaving and easily performed technique, the staircase method was chosen to evaluate the fatigue properties in the present study. Fatigue strength obtained from the staircase method suggests that a half of the control group specimens under the stress level of $30.4 \mathrm{MPa}$ will fracture before $10^{5}$ cycles and the remaining half can survive. In the purified group, such stress level was slightly increased to $31.4 \mathrm{MPa}$. Considering the coefficient of variation (CV; the standard deviation divided by the mean) for the tensile and fatigue strengths, it showed that the CV values of the purified group in two tests were both 
decreased; in the tensile test from 4.4 to $1.0 \%$ and the fatigue test from 6.9 to $4.1 \%$. It suggests that the purified material might be more reliable than the control material under cyclic and static tensile stress modes.

While comparing the fatigue strengths, the increase in the purified group was not significant (from 30.4 to $31.4 \mathrm{MPa}$ ) regardless of the notable improvement in tensile strength (from 36.7 to $40.2 \mathrm{MPa}$ ). Dynamic fatigue behavior is a complex phenomenon, which depends on many factors such as a material's strength and ductility ${ }^{10)}$. Although the tensile strength of the purified group was increased, its ductility decreased simultaneously. According to the results of the purified group, it is suggested that the increase in tensile strength might be compromised by the decrease in ductility. For clarifying the effect of material ductility on fatigue strength, the relative fatigue ratio might be a useful index to indicate the fatigue-resistant ability of each material ${ }^{11)}$. Although the tensile and fatigue tests were conducted under different testing conditions, the relative fatigue ratio of each material was arbitrarily defined as the percentage ratio of cyclic stress amplitude to its tensile strength. After re-calculating the fatigue data in staircase diagrams, the control group showed a significantly higher relative fatigue ratio than that of the purified group $(82.8 \pm 5.6 \mathrm{vs}$ $78.1 \pm 3.1 \%)(p<0.05)$. The comparable fatigue strength and the higher relative fatigue ratio of the control group might be attributed to its plastic deformation and interfacial debonding under repeated tensile stressing.

It can be concluded that by eliminating oxygen, hydroquinone and water from the curing process, the induction period of the polymerization reaction was significantly shortened and its reaction exotherm increased. The deformation-resistance to tensile stress was also improved. Its better bead-matrix interfacial property could be confirmed by the tensile fracture surfaces. However, fracture toughness and fatigue strength were not significantly improved. This might be explained by the different failure mechanisms due to different testing conditions. In general, the polymerization outcomes, mechanical properties and structural reliability of the purified group were improved in this study. While the dental clinicians manipulate the self-curing resin, the storage and curing environments should be kept from oxygen, hydroquinone and water as much as possible.

\section{ACKNOWLEDGEMENTS}

This study was supported by grants (\#14370628 and \#12470404) from the Ministry of Education, Culture, Sports, Science and Technology of Japan.

\section{REFERENCES}

1) Ruyter, I. E.: Release of formaldehyde from denture base polymers, Acta Odontol Scand $38: 17-27,1980$.

2) Ruyter, I. E.: Physical and chemical aspects related to substances related from polymer materials in an aqueous environment, Adv Dent Res 9(4):344-347, 1995.

3) Seki, F., Toida, T. and Nakabayashi N.: Adverse effect of oxygen and water adsorbed by 
PMMA pearls on denture base resin, $J J$ Dent Mater 14(6) :691-696, 1995. (in Japanese)

4) Yang, J.M.: Polymerization of acrylic bone cement using differential scanning calorimetry, Biomaterials 18 : 1293-1298, 1997.

5) Antonucci, J. M. and Toth, E. E.: Extent of polymerization of dental resins by differential scanning calorimetry, J Dent Res 62(2) : 121-125, 1983.

6) Srawley, J.E.: Wide range stress intensity factor expressions for ASTM E-399 standard fracture toughness specimens, Int $J$ Fracture $12: 475-476,1976$.

7) Yamamoto, M. and Takahashi, H.: Tensile fatigue strength of light cure composite resins for posterior teeth, Dent Mater $J$ 14(2) : 175-184, 1995.

8) Fujishima, A. and Ferracane, J. L.: Comparison of four modes of fracture toughness testing for dental composites, Dent Mater 12 : 38-43, 1996.

9) Gilbert, J.L., Ney, D.S. and Lautenschlager, E. P.: Self-reinforced composite poly (methyl methacrylate): static and fatigue properties, Biomaterials 16 : 1043-1055, 1995.

10) Baran, G., Boberick, K. and McCool, J.: Fatigue of restorative materials, Crit Rev Oral Biol Med 12(4): 350-360, 2001.

11) Zardiackas, L. D. and Bayne, S. C.: Fatigue characterization of nine dental amalgams, Biomaterials $6: 49-54,1985$. 